\title{
Association of CD40 -1C/T Polymorphism in the 5'-Untranslated Region with Chronic HBV Infection
}

\author{
Cheng Zhou Xiaoli Jin Jie Tang Jiaqian Fei Chunxia Gu Xi Chen
}

State Key Laboratory for Diagnosis and Treatment of Infectious Diseases, Collaborative Innovation Center for Diagnosis and Treatment of Infectious Diseases, First Affiliated Hospital, College of Medicine, Zhejiang University, Hangzhou, China

\section{Key Words}

CD40-1C/T polymorphism • Asymptomatic HBV carriers • CD40 expression • Soluble CD40 • Immune tolerance

\begin{abstract}
Background: CD40 is an important costimulatory molecule in both celluar and humoral immune responses, involved in the pathogenic processes of chronic inflammatory diseases. Few studies were performed on the association of CD40 single nucleotide polymorphism (SNP) with chronic hepatitis B virus (HBV) infection. In this study, we studied whether the CD40$1 \mathrm{C} / \mathrm{T}$ polymorphism had any effect on the progression of chronic HBV infection in Chinese population. Methods: CD40-1C/T polymorphism in the $5^{\prime}$-untranslated region was analyzed by polymerase chain reaction-restriction fragment length polymorphism (PCR-RFLP) in 453 chronic HBV carriers, who were divided into asymptomatic HBV carriers (ASC), moderate chronic hepatitis B group (MCHB) and severe chronic hepatitis B group (SCHB). 202 healthy individuals in the same region were enrolled in this study as the controls. The CD40 expression on B lymphocytes was detected by flow cytometry. The concentrations of soluble CD40 (sCD40) in sera were assayed by a commercial ELISA kit. Results: Our results showed the frequencies of TT genotype and T allele of CD40-1C/T polymorphism were higher significantly in ASC than those in controls $(P<0.05)$, while this result was not found in either MCHB or $\mathrm{SCHB}$. On the surface of $\mathrm{B}$ lymphocytes, the CD40 expression levels in the individuals with TT genotype were significantly lower than those with CC and CT genotypes in either ASC group or healthy controls $(P<0.001)$. The SCD40 levels in the sera of ASC, MCHB and SCHB groups were significantly higher than the controls $(P<0.001)$. Conclusions: The CD40 $-1 C / T$ polymorphism may contribute to the susceptibility of asymptomatic HBV carriers through its effect on cell-surface CD40 expression, which indicated CD40 signaling was involved in immune tolerance of chronic HBV infection.
\end{abstract}

X. Jin, J. Tang and J. Fei contributed equally to this paper.

Cheng Zhou

KARGER 125
State Key Laboratory of Infectious Disease Diagnosis and Treatment, First Affiliated Hospital of Zhejiang University, No.79, Qingchun Road, Hangzhou 310003, (China) Tel. +86-571-87236447, Fax+86-571-8068731, E-Mail zhoucheng0113@zju.edu.cn 
Zhou et al.: CD40 -1C/T Polymorphism and Chronic HBV Infection

\section{Introduction}

Hepatitis B virus (HBV) infection is the most common cause of liver disease worldwide. More than 350 million persons worldwide have chronic HBV infections. Patients with chronic HBV infection are not only at high risk of progression to liver cirrhosis and hepatocellular carcinoma, but also act as virus reservoir of HBV. The clinical presentations of chronic HBV infection vary from asymptomatic state to fulminant hepatitis [1-3]. Hepatitis B clearance occurs more often in individuals who develop a broad and strong immune response than in those with weak and narrowly focused responses [4]. Many studies indicated that the genetic variants of immune molecules were associated with the body immune status, which is correlated with different clinical outcomes of HBV infections [5-7].

CD40 is a $50-\mathrm{kD}$ cell-surface glycoprotein of the tumor necrosis factor receptor superfamily. It is widely expressed by both immune and non-immune cells, such as B cells, macrophages, dendritic cells (DCs), epithelial, endothelial, hepatocytes, platelets, tumor cells, etc. CD40 interaction with its ligand CD40L promotes DCs to mature and triggers the T-cell activation and differentiation effectively. CD40 ligation of B cells also induces germinal center formation, immunoglobulin isotype switching, somatic hypermutation, which are the key steps for the production of antibodies. Besides, CD40 stimulation also induces the production of different cytokines, chemokines, etc. Therefore, CD40 signaling is crucial in regulating both adaptive immunity (T-cell and $\mathrm{B}$-cell immune responses) and innate immunity [8-10]. Blockade of CD40 signaling results in compromised immune response $[11,12]$. Several studies reported CD40 had been highly expressed in the livers of patients with viral hepatitis as well as hepatocytes infected with HBV virus [13-15], which was correlated with intrahepatic inflammation, necrosis and hepatocyte apoptosis $[16,17]$. A recent report demonstrated that CD40 signaling was able to inhibit replication of hepatitis $\mathrm{C}$ virus in primary human hepatocytes by c-Jun $\mathrm{N}$ terminal kinase activation [18]. Besides, the concentration of sCD40 was also showed to be higher in the sera of patients with liver diseases (including hepatitis B) than those of the controls [19-21]. All these results suggested that $\mathrm{CD} 40 / \mathrm{CD} 40 \mathrm{~L}$ system play an important role in the pathogenesis of viral hepatitis.

CD40-1 C/T polymorphism located in Kozak sequence can influence the initiation of CD40 translation [22], which was reported to be associated with the susceptability of many immune-related, chronic inflammatory diseases such as autoimmune thyroid disease, multiple sclerosis, coronary syndrome, etc [23-26]. But few studies have been found to focus on this polymorphism association with chronic HBV infections. In this paper, we evaluated the association of CD40 -1C/T polymorphism with the susceptibility to disease progression of chronic HBV infection. Moreover, the relationships of this genetic variant with CD40 expression on B lymphocytes and the serum sCD40 level, have been also analyzed in the patients with chronic HBV infection.

\section{Subject and Methods}

\section{Patients and control subjects}

A Total of 453 patients with chronic HBV infection from the First Affiliated Hospital of Zhejiang University (Hangzhou, China) were recruited for the study. The diagnosis of chronic HBV infection was confirmed by seropositivity for HBsAg over 6 months (commercially available enzyme-linked immunosorbent assay kit, Abbott Laboratories, Chicago, IL). Among the subjects, 174 cases were asymptomatic HBV carriers (ASC) with normal serum levels of alanine aminotransferase (ALT) /aspartate aminotransferase (AST) and negative for HBeAg and HBVDNA, without previous history of hepatitis B or any other clinical symptom within 1 year during the study. 165 cases were moderate chronic hepatitis B (MCHB) with previous history of hepatitis $B$ and continuously elevated levels of ALT/AST, but serum total bilirubin (TBil) was less than 10 times of normal upper limit (171 uM) and International Normalized Ratio (INR) less than 1.5, while 114 chronic hepatitis B with TBil more than 10 times of normal upper limit and/or INR exceeds 1.5 were diagnosed as severe chronic hepatitis B (SCHB). Patients with other immune-related diseases or coinfected with other 
Zhou et al.: CD40 -1C/T Polymorphism and Chronic HBV Infection

type hepatitis virus or alcoholic liver disease were excluded. Meanwhile, 202 ethnically matched healthy individuals, who were negative for $\mathrm{HBs} \mathrm{Ag}, \mathrm{HBeAg}, \mathrm{HBeAb}, \mathrm{HBcAg}, \mathrm{HBcAb}$ from the same geographical area were recruited as controls. All subjects were informed consent and the study was approved by the ethics committee of the First Affiliated Hospital of Zhejiang University.

Genotyping of CD40 polymorphisms

Genomic DNA from samples was extracted with the whole blood genomic DNA extraction mini-kit according to the manufacturer's instructions (Hangzhou SIMGEN Biotechnology. Co., Ltd. Hangzhou, China). CD40-1C/T polymorphism was determined by polymerase chain reaction (PCR) and the analysis of restriction fragment lengths polymorphism (RFLP) as previously reported [25]. PCR was performed to amplified the 5'-untranslated region of CD40 using the following primers: 5'-GAAACTCCTGCGCGGTGAAT-3' and 5'-CCTCTTCCC-CGAAGTCTTCC-3' (NCBI Reference Sequence: NC_000020.11). 100ng genomic DNA was amplified in $25 \mathrm{ul}$ reaction containing $0.25 \mathrm{uL}$ of rTaq (Takara Biotechnology CO. LTD., Dalian, China). The PCR procedure is initiated at $95^{\circ} \mathrm{C}$ for $5 \mathrm{~min}$, followed by 30 cycles of $94^{\circ} \mathrm{C}$ for $30 \mathrm{~s}, 60^{\circ} \mathrm{C} 8 \mathrm{C}$ for $30 \mathrm{~s}$, and $72^{\circ} \mathrm{Cfor} 45 \mathrm{~s}$, and extended at $72^{\circ} \mathrm{C}$ for $5 \mathrm{~min}$. Then, $5 \mathrm{ul} \mathrm{PCR}$ product was digested with $5 \mathrm{U}$ of NcoI restriction enzyme (Takara Biotech., Dalian, China) at $37^{\circ} \mathrm{C}$ for $6 \mathrm{~h}$. The digestion products were analyzed on $2.5 \%$ agarose gel. In order to determine the accuracy of the PCR-RFLP method, the results were verified again with direct sequencing in about $10 \%$ randomly selected samples and found no errors.

Flow cytometry

The whole blood from subjects was incubated for an hour at room temperature with PE-conjugated anti-human CD19 (BD Biosciences) and FITC-conjugated anti-human CD40 (invitrogen). FITC-conjugated mouse IgG1 was used as isotype control. Then the blood was hemolyzed and fixed. The cells were analyzed in a Coulter, Epics XL flow cytometer (Beckman Coulter, Germany).

\section{Detection for human sCD40}

Human sCD40 in sera from the subjects were dectected by a commercial ELISA kit (Bender MedSystems Diagnostics GmbH, Vienna, Austria). The procedure was performed according to the kit protocol.

\section{Statistical analysis}

Hardy-Weinberg equilibrium was tested for each polymorphism included in the study with Chisquare test. The frequencies of genotypes and alleles were determined by direct gene counting method and compared by Chi-square test. The associations of the genotype and allele frequencies with chronic HBV infection, were analyzed by unconditional logistic regression after adjusting for the age and gender. Continuous variables among the groups were analyzed by one-way ANOVA. $P$-value less than 0.05 was regarded to be statistically significant. All the statistical analysis was performed by SPSS software version 19.0 (SPSS, Inc., Chicago, IL).

\section{Results}

Clinical and demographic characteristics of population with HBV infection

The baseline and laboratory characteristics in normal controls, ASC, MCHB, and SCHB are summarized in Table 1 . The age was significantly different among the four groups ( $F=2.894$, $P=0.035$, one-way ANOVA). Patients with SCHB were younger than the control and ASC groups (Table $1, P<0.001$ ). As for the gender, more male patients were presented in patients with MCHB and SCHB than those in normal controls (controls vs MCHB, $P<0.05$; controls vs SCHB, $P<0.001$ ), as well as in ASC (ASC vs MCHB, $P<0.05$; ASC vs SCHB, $P<0.001$ ). Also, the percentage of men in SCHB group was higher than that of MCHB $(P<0.05)$. However, there was no significant differences in both the age and gender between the control and ASC groups $(P>0.05)$. The data showed that the age and gender were in good matches between control and ASC groups except MCHB and SCHB groups, indicating the gender and age are sensitive factors related with the different outcomes of HBV infection, which is in accordance with the previous reports $[27,28]$.

\section{KARGER}


Table 1. Characteristics of controls and patients with chronic HBV infection. ACS: asymptomatic HBV carriers; MCHB: moderate chronic hepatitis B; SCHB: severe chronic hepatitis B. ALT: alanine aminotransferase. Compared with controls, ${ }^{*} P<0.05$, ${ }^{* *} P<0.001$. Compared with ASC, $\Delta P<0.05, \Delta \Delta P<0.001$. Compared with MCHB, $\diamond P<0.05$

\begin{tabular}{lllll}
\hline \multirow{2}{*}{ Variables } & \multicolumn{3}{c}{ chronic HBV carriers } \\
\hline Mean age (years) & $44.2 \pm 15.1$ & $44.6 \pm 14.7$ & $43.2 \pm 13.9$ & $40.0 \pm 11.5^{* * \Delta \Delta}$ \\
Male (\%) & $127(62.9)$ & $103(59.2)$ & $123(74.5){ }^{* \Delta}$ & $98(86.7)^{* * \Delta \Delta \Delta}$ \\
HBeAg(+/-) & $0 / 202$ & $0 / 174$ & $87 / 78$ & $82 / 68$ \\
Mean ALT(IU/L) & $22.2 \pm 10.5$ & $23.3 \pm 9.4$ & $272.2 \pm 391.5$ & $461.1 \pm 442.4$ \\
Bilirubin (umol/L) & $12.9 \pm 4.8$ & $12.9 \pm 4.8$ & $55.3 \pm 59.3$ & $291.4 \pm 175.8$ \\
HBVDNA(+/-) & $0 / 202$ & $0 / 174$ & $123 / 42$ & $82 / 32$ \\
\hline
\end{tabular}

Table 2. The distributions of CD40 -1C/T genotype and allele frequencies

\begin{tabular}{|c|c|c|c|c|c|}
\hline \multirow[t]{2}{*}{ groups (n) } & \multicolumn{3}{|c|}{ genotypes (\%) } & \multicolumn{2}{|c|}{ alleles (\%) } \\
\hline & $\mathrm{CC}$ & $\mathrm{CT}$ & $\mathrm{TT}$ & $\mathrm{C}$ & $\mathrm{T}$ \\
\hline Controls (202) & 77 (38.1) & $98(48.5)$ & $27(13.4)$ & $252(62.4)$ & $152(37.6)$ \\
\hline ASC (174) & $51(29.3)$ & 77 (44.3) & $46(26.3)$ & $179(51.4)$ & $169(48.6)$ \\
\hline МCHB (165) & $61(40.0)$ & $73(44.2)$ & 31 (18.8) & 195 (59.1) & 135 (40.9) \\
\hline SCHB (114) & $43(37.7)$ & $56(49.1)$ & $15(13.2)$ & $142(62.3)$ & $86(37.7)$ \\
\hline$\chi^{2}$ & 13.968 & & & 11.043 & \\
\hline$P$ & 0.03 & & & 0.011 & \\
\hline
\end{tabular}

Table 3. The comparisons of CD40 -1C/T genotype and allele frequencies among four groups. ${ }^{*} P<0.05$

\begin{tabular}{lllll}
\hline & \multicolumn{2}{c}{ genotype } & \multicolumn{2}{c}{ Allele } \\
& $\chi 2$ & $P$ & $\chi^{2}$ & $P$ \\
\hline ASC vs controls & 10.721 & $0.005^{*}$ & 9.145 & $0.002^{*}$ \\
ASC vs MCHB & 3.685 & 0.158 & 4.012 & $0.045^{*}$ \\
ASC vs SCHB & 7.580 & $0.023^{*}$ & 6.565 & $0.010^{*}$ \\
MCHB vs controls & 2.077 & 0.345 & 0.823 & 0.364 \\
MCHB vs SCHB & 1.654 & 0.437 & 0.574 & 0.449 \\
SCHB vs controls & 0.011 & 0.995 & 0.001 & 0.981 \\
\hline
\end{tabular}

Distribution of CD40-1C/T genotype and allele frequencies

The genotype distributions for CD40-1C/T polymorphism were in Hardy-Weinberg equilibrium in each group $(P>0.05)$. The genotype and allele frequencies of this CD40 SNP in patients and control groups are summarized in Table 2 and 3. There were significant differences in the distributions of the genotypes and alleles among the four groups (Table 2, for genotypes, $\chi^{2}=13.968, P=0.03$; for alleles, $\chi^{2}=11.043, P=0.011$ ). As shown in Table 3 , the frequency of the CD40 TT genotype was significantly higher in ASC group than the control and SCHB groups (ASC vs controls, $P=0.005$; ASC vs SCHB, $P=0.023$ ), and the T allele frequency of ASC group was also significantly higher than the control, MCHB and SCHB groups (ASC vs controls, $P=0.002$; ASC vs MCHB, $P=0.045 ;$ ASC vs SCHB , $P=0.010$ ). However, neither genotype nor allele frequency showed significant differences among the control, MCHB and SCHB groups. Moreover, we analyzed the data by nonconditional logistic regression after adjustment with age and sex. The results showed that the subjects with TT genotype had an KARGER 
Table 4. The association test for CD40-1C/T genotypes between asymptomatic HBV carriers and controls. The data were performed by logistic regression analysis adjusted with age and sex. ${ }^{\mathrm{a}, \mathrm{b}, \mathrm{c}} \mathrm{Comparison}$ between control and ASC groups. ${ }^{\mathrm{a} C o m p a r i s o n ~ C C ~ w i t h ~ C T+T T . ~}{ }^{\mathrm{b}}$ Comparison CC+CT with TT. ${ }^{\mathrm{c} C o m p a r i s o n} \mathrm{CC}$ with CT. OR: odds rate, $\mathrm{CI}$ : confidence intervals

\begin{tabular}{|c|c|c|c|c|c|c|c|c|}
\hline \multirow[t]{2}{*}{ Genotypes } & \multirow{2}{*}{$\begin{array}{l}\text { ASC } \\
(n=174)\end{array}$} & \multirow{2}{*}{$\begin{array}{l}\text { Controls } \\
(n=202)\end{array}$} & \multicolumn{2}{|c|}{ Dominant modela } & \multicolumn{2}{|c|}{ Recessive model ${ }^{\mathrm{b}}$} & \multicolumn{2}{|c|}{ Additive modelc } \\
\hline & & & $P$ & $\mathrm{OR}(95 \% \mathrm{CI})$ & $P$ & OR(95\%CI) & $P$ & OR(95\%CI) \\
\hline \multirow[t]{2}{*}{$\mathrm{CC}$} & 51 & 77 & & & & & & \\
\hline & $(29.3 \%)$ & $(38.1 \%)$ & 0.169 & 1.338 & 0.002 & 2.278 & 0.005 & 2.212 \\
\hline \multirow[t]{2}{*}{ CT } & 77 & 98 & & $(0.883-2.033)$ & & (1.354-3.833) & & $(1.267-3.861)$ \\
\hline & $(44.3 \%)$ & $(48.5 \%)$ & & & & & & \\
\hline \multirow[t]{2}{*}{ TT } & 46 & 27 & & & & & & \\
\hline & (26.3\%) & (13.4\%) & & & & & & \\
\hline
\end{tabular}

Fig.1. CD40 expressions on B cells in the control and ASC groups with different genotypes of CD40 -1 C/T polymorphism. CD40 membrane expression on B cells were detected by flow cytometry and the obtained data were analyzed by one-way ANOVA. Compared with CC genotype in corresponding group, ${ }^{*} P<0.05$, ${ }^{* *} P<0.001$, Compared with CT genotype in corresponding group, $\Delta P<0.001$.

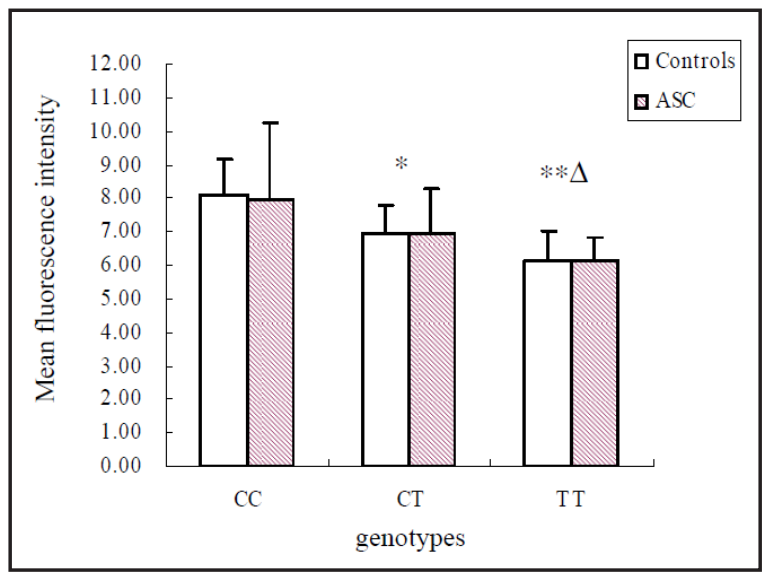

increased susceptibility to asymptomatic HBV carriers compared to those with at least $1 \mathrm{C}$ allele (for recessive model, OR 2.278; 95\% CI, 1.354-3.833; $P=0.002$, and for additive model, OR 2.212; 95\% CI, 1.267-3.861; $P=0.005$, shown in Table 4). Thus, genetic variation of CD40 $-1 \mathrm{C} / \mathrm{T}$ polymorphism was strongly associated with susceptibility to asymptomatic carriers after HBV infection.

Effect of the CD40-1C/T polymorphism on CD40 expression on B lymphocytes

B lymphocytes are the main cells that express CD40 on their surface. They are also the antibody-produced cells in adaptive immune response. The CD40-1C/T SNP is located in the Kozak consensus sequence of CD 40 gene, which plays a key role for CD 40 translation [22]. In order to study whether the CD40-1C/T polymorphism has any effect on the CD40 expression in these subjects and whether there are any differences of CD40 expression between control and ASC groups, we detected the membrane CD40 expression on B lymphocytes by flow cytometry in 94 asymptomatic HBV-carriers and 77 healthy controls. Our data showed the membrane CD40 expressions on B lymphocyte were not different significantly between control and ASC groups [mean fluorescence intensity (MFI), 7.12 \pm 1.18 vs $7.09 \pm 1.73$, $P>0.05]$. However, there are significant differences in CD40 membrane expressions of $B$ lymphocyte among CC, CT and TT genotypes in both control and ASC groups $(P<0.001$, oneway ANOVA.). As shown in Fig. 1, the highest expression of membrane CD40 on B cells existed in the individuals with CC genotype when compared to those with CT or TT genotype (CC vs CT, $P<0.05$; CC vs TT, $P<0.001$ ) in both groups, and the individuals with CT genotype also showed higher levels of CD40 expression than those with TT genotype ( CT vs TT, $P<0.05$ ). Thus, the CD40 -1C/T polymorphism has regulatory effects on CD40 membrane expression, which might affect the strength of CD40 signaling in both cellular and humoral immune responses. 
Fig. 2. Comparison of serum sCD40 levels in different groups of subjects. Serum sCD40 were detected in sera of subjects and the obtained data were logarithmically transformed and then analyzed by one-way ANOVA. Compared with healthy controls. ${ }^{*} P<0.001$.

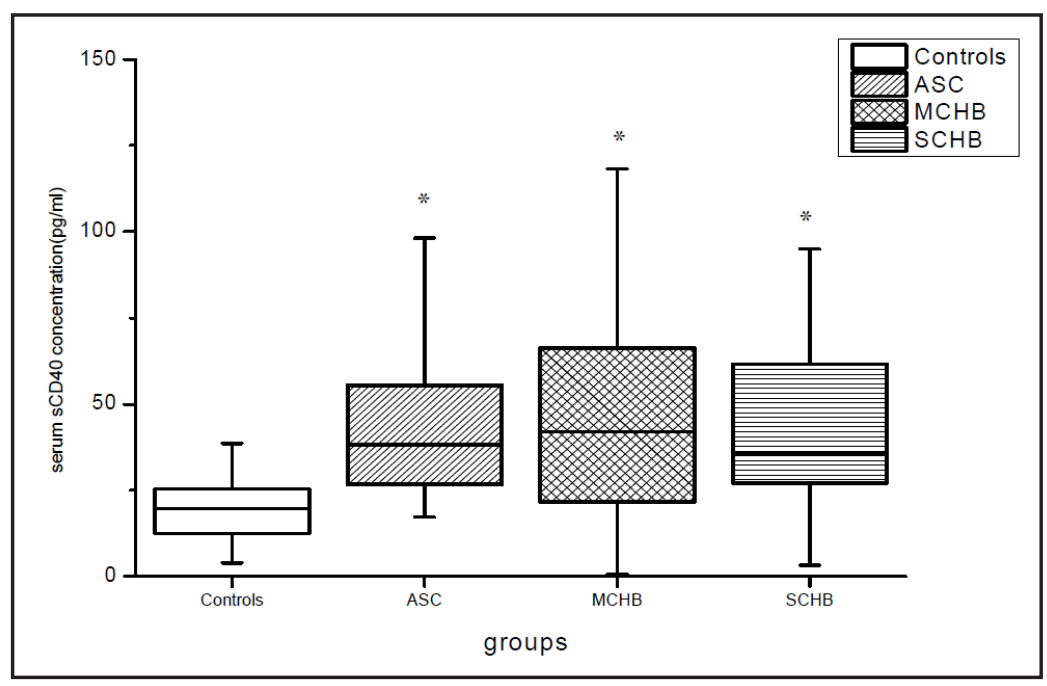

sCD40 levels in chronic HBV infection and its relationship with CD40-1C/T polymorphism

It was reported that serum sCD40 levels was significantly higher in patients with liver diseases including chronic viral hepatitis [19-21], and the renal function is a key factor in determining the sCD40 level [29]. Thus, in this study we have detected serum sCD40 concentrations in 76 controls and 214 cases with chronic HBV infection (96 ASC, 80 MCHB and 38 SCHB), all of whom have normal renal function. Similar results were also obtained in our study to confirm that serum sCD40 levels in chronic hepatitis B (including MCHB and SCHB) are significantly higher than those of the controls. Besides, we also found higher levels of serum sCD40 presented in asymptomatic HBV carriers (Fig. 2, $P<0.001$ ). However, there are no significant differences among ASC, MCHB and SCHB groups. Then, we are wondering whether there is any association between the serum levels of SCD40 and CD40$1 \mathrm{C} / \mathrm{T}$ polymorphism. The results showed the serum levels of sCD40 are not associated with CD40-1C/T genotypes in either chronic HBV infections or the healthy controls (data not shown). Since sCD40 is an important negative molecule in CD40 signaling, the above results suggested CD40 pathway might be involved in immune tolerance of chronic HBV infections, and CD40 -1C/T polymorphism have no effect on the expressions of the soluble form of CD40 in sera.

\section{Discussion}

It was estimated that about $70 \%$ of chronic HBV-infected individuals were asymptomatic inactive HBV carriers because of immune tolerance. Previous data showed about $10 \%$ of asymptomatic HBV carriers occurred liver cirrhosis, and/or even hepatocellular carcinoma after about 20 years. Persons with asymptomatic chronic HBV infection should be paid high attention and followed up continuously [3]. Therefore, researches on the mechanism about how the asymptomatic HBV carriers occur should be of great significance from both healthy and economic aspects. It is well-known that the clinical outcome of HBV infection depends on the interaction between viral replication and host immunity, and the genetic background was one of the most key factors that influence the host immunity [4-6]. But limited researches were reported on the genetic factors which influence the genesis of asymptomatic chronic HBV infections. Previous studies have demonstrated that activation of CD40 signaling is indispensable for the induction of effective virus-specific CD8+ T-cell responses, which is required for the virus clearance [30,31]. Using a transgenic mouse model, Chisari et al. showed that the HBV antigen-specific CD8+ T cell exhaustion was able to be rescued by CD40mediated mDC activation [32]. Thus, CD40 signaling plays critical roles in antiviral immune responses. The diversity of CD40 expression on cell surface is one of the major factors that 
regulate the intensity of CD40 signaling. Among all the SNPs of CD40, $-1 \mathrm{C} / \mathrm{T}$ polymorphism, located at the KOZAK region of CD40 gene, is the only one SNP which is known to influence the CD40 expression. In the present study, we found the CD40-1C/T polymorphism was associated with increased susceptibility to asymptomatic HBV carriers. The T allele and TT genotype frequencies were found significantly higher in asymptomatic HBV carriers. The CD40 -1C/T polymorphism was able to regulate the expression levels of membrane CD40 on B cells, since our results showed there were significant differences of CD40 membrane expressions among the individuals with CC, CT and TT genotypes. Besides, compared with the healthy controls, the serum sCD40 increased in chronic HBV infections, including ASC, MCHB and SCHB patients, but its levels were not correlated with CD40 -1C/T polymorphism. To our knowledge, this is the first report that the genetic variants of CD40 gene are correlated with an individual's susceptibility to asymptomatic chronic HBV infection.

It is demonstrated that the SNP located in the Kozak sequence is able to influence the initiation rate of a gene translation [22]. In this study, we showed that the CD40 - $1 \mathrm{C} / \mathrm{T}$ polymorphism can cause the alteration of CD40 translation on human B cells. The expression of CD40 in the individuals with TT genotype was significantly lower than those with CC and CT genotypes in both ASC and control groups, which is agreement with other studies [25, 33]. Our findings that the frequency of TT genotype was significantly higher in ASC groups might be understood as follows: TT genotype of CD40-1C/T polymorphism decreases CD40 expression on cell surface as we and others confirmed. Then the decreased CD40 expression down-regulates both viral-specific and innate immune responses via CD40 signaling, which in turn results in the host immune tolerance, the main immune characteristic of asymptomatic chronic HBV carriers.

Soluble form of CD40 was demonstrated to act as an agonist of CD40/CD40L interaction. Since its interaction with CD40 was able to reduce immunoglobulin production and T cell activation, this soluble molecule was supposed to have an immunosuppressive effect [34]. In $\mathrm{HBV}$ infections, this soluble form of CD40 was found significantly higher in patients with acute and chronic HBV hepatitis, which was correlated with ALT levels, indicating there should be association between SCD40 levels and liver inflammations [20,21]. However, no data of the sCD40 concentrations were reported in the asymptomatic chronic HBV carriers. Here we showed that SCD40 was also significantly higher than those of healthy controls although the serum aminotransferases were normal in their bodies. The mechanism of increased sCD40 level in ASC is still elusive. It was reported that SCD40 was produced by either alternative splicing or the proteolytic cleavage of membrane-anchored CD40 by a tumor necrosis factorconverting enzyme (TACE) [35-37]. In liver diseases, Schmilovitz-Weiss et al. suggested that sCD40 probably derive from the liver [19]. Other studies in chronic HCV infection also found sCD40 was higher significantly than healthy controls, suggested that the virus might be able to stimulate the sCD40 formation resulting in the increased levels of sCD40 [38]. In our study, we found the lowest expression of membrane CD40 on B cells existed in the individuals with TT genotype compared with CC or CT genotype in both control and ASC groups (shown in Fig. 1) and the frequency of the CD40 TT genotype was significantly higher in ASC group. So, it seems unlikely that the elevated circulating SCD40 in ASC group is the proteolytic cleavage of membrane CD40 by TACE. Thus, in our opinion in ASC group, HBV might enhance the synthesis of sCD40 probably by either alternative splicing or an unknown signaling, which needs further study. However, it is not difficult for us to understand this result. Since sCD40 binds with membrane CD40 to counteract CD40/CD40L interaction, the increased sCD40 levels in ASC subjects are supposed to inhibit CD40 signaling, inducing the immunosuppressive status of ASC. Based on the previous finding that CD40 -1C/T polymorphism was able to influence the levels of sCD40L by feedback mechanism via its regulation on the CD40 expression [39], we also want to know if there is any effect of this CD40 SNP on the regulation of SCD40 levels and the results showed there was no relationship between the SCD 40 concentrations and CD $40-1 \mathrm{C} / \mathrm{T}$ genotypes, indicating SCD40 expression might not be influenced by this CD40 SNP. Because of the relative small number of samples assayed in this study, more data are needed to confirm the result.

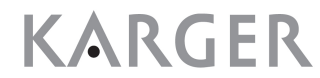


In conclusion, our study first revealed the association of CD40-1C/T polymorphism with susceptibility to asymptomatic chronic HBV carriers, and the increased serum levels of sCD40 in these carriers, which suggested that the strength of CD40 signaling should be one of the most important pathways involved in host immune tolerance after HBV infection. This naturally occurring genetic variant of CD40 may have effects on the pathogenesis of chronic HBV infection by its regulation of CD40 expression, which might be useful in predicting the clinical outcomes of HBV infection.

\section{Acknowledgments}

We thank Dr. Ge C for her excellent technical assistance. This work was supported by National Natural Science Foundation of China (No. 81273321).

\section{References}

1 Trepo C, Chan HL, Lok A: Hepatitis B virus infection. Lancet DOI: 10.1016/S0140-6736(14)60220-8.

2 Chang ML, Liaw YF: Hepatitis B flares in chronic hepatitis B: pathogenesis, natural course and management. J Hepatol. DOI: 10.1016/j.jhep.2014.08.033.

3 Amarapurkar D: Asymptomatic HBsAg carrier: A Case for Concern; in Banerjee AK, Bandyapadhyay S (eds): Med Update, Mumbai, Assoc Physici India, 2011, vol 21, pp 358-363.

4 Knolle PA, Thimme R: Hepatic immune regulation and its involvement in viral hepatitis infection. Gastroenterology 2014;146: 193-1207.

Zeng Z: Human genes involved in hepatitis B virus infection. World J Gastroenterol 2014;20:7696-7706. Thio CL, Astemborski J, Bashirova A, Mosbruger T, Greer S, Witt MD, Goedert JJ, Hilgartner M, Majeske A, O'Brien SJ, Thomas DL, Carrington M: Genetic protection against hepatitis B virus conferred by CCR5 Delta32: Evidence that CCR5 contributes to viral persistence. J Virol 2007;81:441-445.

7 Deng G, Zhou G, Zhang R, Zhai Y, Zhao W, Yan Z, Deng C, Yuan X, Xu B, Dong X, Zhang X, Zhang X, Yao Z, Shen Y, Qiang B, Wang Y, He F: Regulatory polymorphisms in the promoter of CXCL10 gene and disease progression in male hepatitis B virus carriers. Gastroenterology 2008;134:716-726.

8 Elgueta R, Benson MJ, de Vries VC, Wasiuk A, Guo Y, Noelle RJ: Molecular mechanism and function of CD40/ CD40L engagement in the immune system. Immunol rev 2009;229:152-172.

-9 Zhang K, Zhang L, Zhu D, Bae D, Nel A, Saxon A. CD40-mediated p38 mitogen-activated protein kinase activation is required for immunoglobulin class switch recombination to IgE. J Allergy Clin Immunol 2002;110:421-428.

10 Danese S, Sans M, Fiocchi C: The CD40/CD40L costimulatory pathway in inflammatory bowel disease. Gut 2004;53:1035-1043.

11 Merrill JT: Co-stimulatory molecules as targets for treatment of lupus. Clin Immunol 2013; 148: 369-375.

12 Pilat N, Schwarz C, Wekerle T: Modulating T-cell costimulation as new immunosuppressive concept in organ transplantation. Curr Opin Organ Transplant.2012;17:368-375.

13 Tang Y, Chen Y, Ni B, Yang D, Guo S, Wu Y: Up-regulation of the expression of costimulatory molecule CD40 in hepatocytes by hepatitis B virus x antigen. Biochem Bioph Res Co 2009;384:12-17.

14 Leifeld L, Trautwein C, Dumoulin FL, Manns MP, Sauerbruch T, Spengler U: Enhanced expression of CD80 (B7-1), CD86 (B7-2), and CD40 and their ligands CD28 and CD154 in fulminant hepatic failure. Am J Pathol 1999;154:1711-1720.

15 Shiraki K, Sugimoto K, Okano H, Wagayama H, Fujikawa K, Yamanaka T, Ito T, Ohmori S, Sakai T, Takase K, Nakano T: CD40 expression in HCV-associated chronic liver diseases. Int J Mol Med 2006;18:559-563.

16 Yan J, Jie Z, Hou L, Wanderley JL, Soong L, Gupta S, Qiu S, Chan T, Sun J: Parenchymal expression of CD40 exacerbates adenovirus-induced hepatitis in mice. Hepatology 2011;53:1455-1467.

17 Zhou F, Ajuebor MN, Beck PL, Le T, Hogaboam CM, Swain MG: CD154-CD40 interactions drive hepatocyte apoptosis in murine fulminant hepatitis. Hepatology 2005;42:372-380.

18 Rau SJ, Hildt E, Himmelsbach K, Thimme R, Wakita T, Blum HE, Fischer R: CD40 inhibits replication of hepatitis $\mathrm{C}$ virus in primary human hepatocytes by c-Jun $\mathrm{N}$ terminal kinase activation independent from the interferon pathway. Hepatology 2013;57:23-36. 
Zhou et al.: CD40 -1C/T Polymorphism and Chronic HBV Infection

-19 Schmilovitz-Weiss H, Belinki A, Pappo O, Sulkes J, Melzer E, Kaganovski E,Kfir B, Tur-Kaspa R, Klein T, BenAri Z: Role of circulating soluble CD40 as an apoptotic marker in liver disease. Apoptosis 2004;9:205-210.

-20 Shen HH, Sun HY, Zhao JM, Hou J, Bai BK, Hu Y, Kong W, Mao PY: Level and clinical significance of soluble CD40 in patients with chronic hepatitis B. Zhonghua Shi Yan He Lin Chuang Bing Du Xue Za Zhi 2008;22:373-375. Chinese

21 Liu H, Qi CJ, Zhuang YM, Gan JH, Li HL, Yin CS, Zhang XG: Serum levels and clinical significance of soluble CD40 in liver disease. Xi Bao Yu Fen Zi Mian Yi Xue Za Zhi. 2006;22:738-741. Chinese.

22 Kozak M: Regulation of translation via mRNA structure in prokaryotes and eukaryotes. Gene 2005;361:1337.

23 Inoue N, Watanabe M, Yamada H, Takemura K, Hayashi F, Yamakawa N, Akahane M,Shimizuishi Y, Hidaka Y, Iwatani Y: Associations between autoimmune thyroid disease prognosis and functional polymorphisms of susceptibility genes, CTLA4,PTPN22, CD40, FCRL3 and ZFAT, previously revealed in genome-wide association studies. J Clin Immunol 2012;32:1243-1252.

24 Sokolova EA, Malkova NA, Korobko DS, Rozhdestvenskii AS, Kakulya AV, Khanokh EV, Delov RA, Platonov FA, Popova TY, Aref' eva EG, Zagorskaya NN, Alifirova VM, Titova MA, Smagina IV, El' chaninova SA, Popovtseva AV, Puzyrev VP, Kulakova OG, Tsareva EY, Favorova OO, Shchur SG, Lashch NY, Popova NF, Popova EV, Gusev EI,Boyko AN, Aulchenko YS, Filipenko ML: Association of SNPs of CD40 gene with multiple sclerosis in Russians. PLoS One 2013;8:e61032.

25 Wang M, Li Y, Li W, Xia ZE, Wu Q: The CD40 gene polymorphism rs1883832 is associated with risk of acute coronary syndrome in a Chinese case-control study. DNA Cell Biol 2011;30:173-178.

-26 Yan J, Wang C, Du R, Liu P, Chen G: Association analysis of CD40 gene polymorphism with acute coronary syndrome. Clin Exp Med 2010;10:253-258.

27 Chen YM, Yu CP, Wan SY, Shen YJ, Lin DY: Impact of gender, viral transmission and aging in the prevalence of hepatitis B surface antigen. Chang Gung Med J 2009;32:155-164.

28 Durazzo M, Belci P, Collo A, Prandi V, Pistone E, Martorana M, Gambino R, Bo S: Gender specific medicine in liver diseases: a point of view. World J Gastroenterol. 2014;20:2127-2135.

-29 Esposito P, Rampino T, Gregorini M, Gabanti E, Bianzina S, Dal Canton A: Mechanisms underlying sCD40 production in hemodialysis patients. Cell Immunol. 2012;278:10-15.

30 Bourgeois C, Rocha B, Tanchot C: A role for CD40 expression on CD8+ T cells in the generation of CD8+ T cell memory. Science 2002;297:2060-2063.

-31 Bachmann MF, Hunziker L, Zinkernagel RM, Storni T, Kopf M: Maintenance of memory CTL responses by T helper cells and CD40-CD40 ligand: antibodies provide the key. Eur J Immunol 2004;34:317-326.

-32 Isogawa M, Chung J, Murata Y, Kakimi K, Chisari FV: CD40 activation rescues antiviral CD8 ${ }^{+} \mathrm{T}$ cells from PD1-mediated exhaustion. PLOS Pathog 2013;9:e1003490.

33 Jacobson EM, Concepcion E, Oashi T, Tomer Y: A Graves' disease-associated Kozak sequence singlenucleotide polymorphism enhances the efficiency of CD40 gene translation: a case for translational pathophysiology. Endocrinology 2005;146:2684-2691.

-34 Esposito P, Rampino T, Dal Canton A: Soluble CD40 as a modulator of CD40 pathway. Immunol Lett 2012;147:85-86.

35 Tone M, Tone Y, Fairchild PJ, Wykes P, Waldmann H: Regulation of CD40 function by its isoforms generated through alternative splicing, Proc Natl Acad Sci USA 2001;98:1751-1756.

-36 Eshel D, Toporik A, Efrati T, Nakav S, Chen A, Douvdevani A: Characterization of natural human antagonistic soluble CD40 isoforms produced through alternative splicing, Mol Immunol 2008;46:250-257.

37 Contin C, Pitard V, Itai T, Nagata S, Moreau JF, Déchanet-Merville J: Membrane-anchored CD40 is processed by the tumor necrosis factor-alpha-converting enzyme. Implications for CD40 signaling. J Biol Chem 2003;278:32801-32809.

- 38 Lapiński TW, Pogorzelska J, Grzeszczuk A, Swiderska M, Kowalczuk O, Nikliński J, Flisiak R: The importance of sCD40 and SCD40L concentration in patients with chronic HCV infection and HIV co-infection. Przegl Epidemiol 2014;68:11-15, 105-108.

-39 Zhang B, Wu T, Song C, Chen M, Li H, Guo R: Association of CD40-1C/T polymorphism with cerebral infarction susceptibility and its effect on SCD40L in Chinese population. Int Immunopharmacol 2013;16:461-465. 